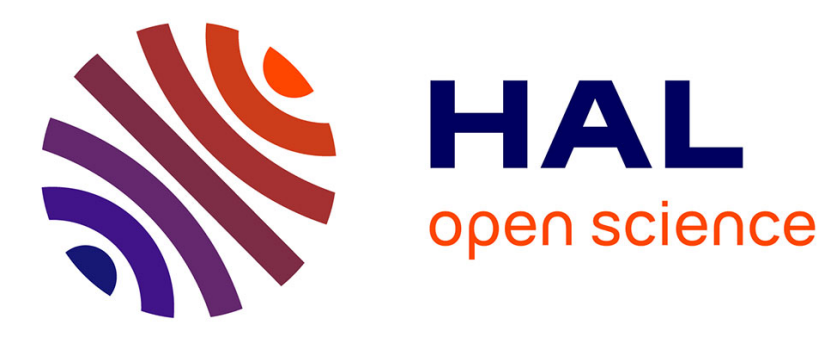

\title{
Using watershed and multimodal data for vessel segmentation: Application to the superior sagittal sinus
}

Nicolas Passat, Christian Ronse, Joseph Baruthio, Jean-Paul Armspach, Jack Foucher

\section{- To cite this version:}

Nicolas Passat, Christian Ronse, Joseph Baruthio, Jean-Paul Armspach, Jack Foucher. Using watershed and multimodal data for vessel segmentation: Application to the superior sagittal sinus. International Symposium on Mathematical Morphology (ISMM), 2005, Paris, France. pp.419-428, 10.1007/1-4020-3443-1_38. hal-01694973

\section{HAL Id: hal-01694973 \\ https://hal.univ-reims.fr/hal-01694973}

Submitted on 26 Feb 2018

HAL is a multi-disciplinary open access archive for the deposit and dissemination of scientific research documents, whether they are published or not. The documents may come from teaching and research institutions in France or abroad, or from public or private research centers.
L'archive ouverte pluridisciplinaire HAL, est destinée au dépôt et à la diffusion de documents scientifiques de niveau recherche, publiés ou non, émanant des établissements d'enseignement et de recherche français ou étrangers, des laboratoires publics ou privés. 


\title{
USING WATERSHED AND MULTIMODAL DATA FOR VESSEL SEGMENTATION: APPLICATION TO THE SUPERIOR SAGITTAL SINUS
}

\author{
N. Passat ${ }^{1,2}$, C. Ronse ${ }^{1}$, J. Baruthio ${ }^{2}$, J.-P. Armspach ${ }^{2}$, J. Foucher ${ }^{3}$ \\ ${ }^{1}$ LSIIT, UMR 7005 CNRS-ULP, Strasbourg I University, France \\ ${ }^{2}$ IPB, UMR 7004 CNRS-ULP, Strasbourg I University, France \\ ${ }^{3}$ U405 INSERM Strasbourg I University, France
}

\begin{abstract}
Magnetic resonance angiography (MRA) provides 3-dimensional data of vascular structures by finding the flowing blood signal. Classically, algorithms dedicated to vessel segmentation detect the cerebral vascular tree by only seeking the high intensity blood signal in MRA. We propose here to use both cerebral MRA and MRI and to integrate a priori anatomical knowledge to guide the segmentation process. The algorithm presented here uses mathematical morphology tools (watershed segmentation and grey-level operators) to carry out a simultaneous segmentation of both blood signal in MRA and blood and wall signal in MRI. It is dedicated to the superior sagittal sinus segmentation but similar strategies could be considered for segmentation of other vascular structures. The method has been performed on 6 cases composed of both MRA and MRI. The results have been validated and compared to other results obtained with a region growing algorithm. They tend to prove that this method is reliable even when the vascular signal is inhomogeneous or contains artefacts.
\end{abstract}

Keywords: vessel segmentation, watershed segmentation, a priori knowledge, MRA, MRI

\section{Introduction}

Magnetic resonance angiography (MRA) is a technique [5] frequently used to provide $3 \mathrm{D}$ images of cerebral vascular structures. The availability of precise information about brain vascular networks is fundamental for planning and performing neurosurgical procedures, but also for detecting pathologies such as aneurysms and stenoses. Since all classical image processing tools have been applied more or less successfully to the case of vessel segmentation, it might be interesting to explore new kinds of algorithms involving a priori knowledge. In a previous paper [8] we proposed a first attempt to use anatomical knowledge as a way to guide a segmentation algorithm. A major 
breakthrough of this work was the creation of an atlas dividing the head into different areas presenting homogeneous vessel properties. The use of this atlas enables to store a priori knowledge concerning the vessels located in each area and then to propose ad hoc segmentation algorithms. In this paper, we propose an algorithm dedicated to one of these areas, containing a main vessel of the venous tree: the superior sagittal sinus (SSS). This algorithm is based on mathematical morphology tools (watershed segmentation and grey-level operators). It also integrates a priori anatomical knowledge and uses both MRA and MRI data in order to take advantage of both acquisition techniques. It uses a multiresolution slice by slice process, simultaneously segmenting the flowing blood signal in MRA and the blood and vessel wall in MRI. This paper is organized as follows. In Section 2, we review previous approaches concerning vessel segmentation. In Section 3, we describe the way to use anatomical knowlege. In Section 4, the proposed algorithm is described. In Section 5, technical details concerning the method and the database used for validation are provided. In Section 6, the method is tested and compared to a region growing algorithm. Discussion and projects are presented in Section 7.

\section{Related work}

The vessel segmentation methods can be divided into several categories, corresponding to the main strategies used to carry out the segmentation. The first proposed strategies were based on filtering [4]. Method based on mathematical morphology (hysteresis thresholding in [7], grey level erosions and dilations in [3] or grey-scale skeletonization in [10]) and region growing [11] have also been proposed. More recently, methods based on vessel tracking [6], and crest line detection [1] have also been proposed.

It has to be noticed that very few vessel segmentation methods have been designed to process multimodal data. A method proposed in [9] for cerebral vascular structures visualization, uses both 3D MRA and 2D X-ray images. A method has been proposed by us in [8], where angiographic and non angiographic data are involved in an atlas-based region growing algorithm. Nevertheless, the simultaneous use of images from different modalities is quite unusual. The algorithm presented here, based on watershed [2] segmentation and mathematical morphology operators, proposes to uses both MRA and MRI to take advantage of anatomical knowledge concerning the brain superficial venous structures.

\section{A priori knowledge integration}

The SSS presents many invariant properties (i.e. properties being identical for every subjects) which can be useful for guiding a segmentation process. These properties and a way to use them are described as follows. 
Trajectory properties: A way to guide normal planes computation. The regular trajectory of the SSS and its position relatively to the cerebral median plane and the surface of the head theoretically enable to compute successive planes being perpendicular to the sinus axis. Indeed, if the surface of the head and the sagittal median plane of the brain can be found, then their intersection provides a curve. A normal plane computation using the points of that curve finally gives planes being normal to the sinus axis too. Using that strategy, it becomes possible to perform a segmentation of the sinus slice by slice.

Structures intensity and relative positions: A way to guide watershed segmentation. Observing MRA slices (Figure 1, right lower pictures), one can see that only the flowing blood is generally quite visible as it presents the highest intensity. In MRI, more structures can be observed in an easier way (Figure 1 , right upper pictures). Although the flowing blood does not present a very high intensity, it can be observed surrounded by the dura mater. The brain hemispheres present a nearly identical intensity, such as a part of the skull. These four structures are separated by areas of low intensity and their relative positions are globally invariant.

MRA and MRI intensity properties can then be used to perform watershed segmentation on slices of the sinus region. Indeed, a gradient computation on the MRA should correctly delineate the blood from the remaining structures. A watershed segmentation could also be directly used on the MRI images to segment the different structures (considering low intensity regions as the frontier between them). Since the main problem of watershed segmentation remains oversegmentation, it is important to choose correct markers to initialize it. This could be done here by sharing information between MRA and MRI segmentation (the segmentation of blood in MRA could be used to find a marker for the dura mater in MRI, and vice versa) or by sharing information between successive MRI or MRA segmentations.

Structure homogeneity along the sinus trajectory: Justifying an iterative slice by slice strategy. The sinus and its neighboring structures present quite invariant position properties. By observing slices at different points on the SSS trajectory, we can also observe that their size and distance from each other are different (Figure 1 right pictures) but vary smoothly. This property could be efficiently used to start from one slice and successively generate markers to initialize segmentation of the neighbor slices. This is generally done in vessel tracking algorithms. A weakness of the vessel tracking approach is that a segmentation error in one slice will generally have consequences on all the following ones. In order to avoid such problems, an alternative could be to propose an iterative approach. For each slice, it consists in starting, from an average image of the current slice and its neighbors. A segmentation of this 

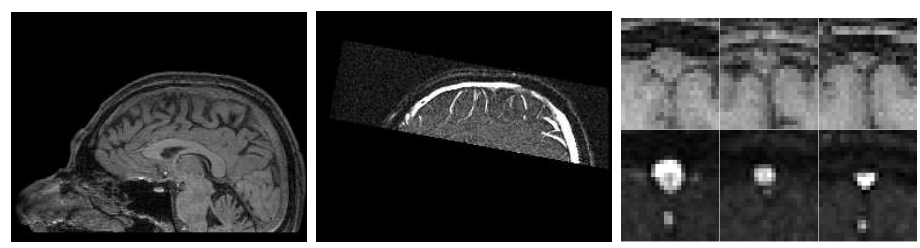

Figure 1. Left: T1 MRI sagittal slice of the whole head. Middle: MRA sagittal slice of the top of the head. Right: slices of MRA and MRI data at various positions on the SSS.

average slice can then be used to generate markers for a new segmentation of a new average slice, closer from the current slice. This process can then be iterated until segmenting the real slice.

\section{Method}

Input and output. The method takes as input a classical MRI and a MRA of the same patient. They must contain at least the top of head and have to be correctly superimposed. If they are not, they can be superimposed by performing a rigid registration (using translations and rotations). Figure 1 illustrates an example of such data. The method provides two resulting images: a segmentation of the flowing blood detected in the MRA and a segmentation of the dura mater surrounding the blood and then forming the sinus wall, from the MRI.

Preprocessing. The segmentation process is not carried out on the global images but on slices that must be normal to the sinus axis. A first step then consists in computing these slices. The sinus axis is parallel to a curve obtained by intersecting the cerebral sagittal median plane and the head surface. The surface of the head can be easily found by thresholding, while the sagittal median plane of the brain can be found by an histogram analysis of the MRA image. The intersection of that plane and the surface of the head then provides a discrete curve. A normal vectors computation on the points of the curve finally enables to compute planes being normal to the curve and also to the sinus axis.

That step then provides two sets of slices of the SSS. The first set is composed of MRI slices while the second contains MRA slices, the $n$-th slice of the first set corresponding to the $n$-th slice of the second. It has been experimentally observed that sets of 256 slices were sufficient to carry out the segmentation. Moreover, we sample the slices to keep $23 \times 26$ voxel-slices located 11 $\mathrm{mm}$ away from the head surface, assuming that small slices centered on the sinus and containing neighboring structures enable to obtain correct results with a lower computation time. 
Definitions and notations. In the following, a slice $s$ of $n \times m$ voxels will be considered as a function of $[0, n-1] \times[0, m-1] \rightarrow \mathbb{N}$. We will always assume that $\alpha \in\{m r a, m r i\}$. Let $\left\{s_{i}^{m r a}\right\}_{i=0}^{t}$ and $\left\{s_{i}^{m r i}\right\}_{i=0}^{t}$ be sequences of MRA and MRI slices. It has to be noticed that for any $a, b, c \in[0, t]$ such that $a<b<c$ the slice $s_{b}^{\alpha}$ is physically located between $s_{a}^{\alpha}$ and $s_{c}^{\alpha}$. For each $i \in[0, t]$ let $\left\{I_{k}^{i}\right\}_{k=0}^{\omega}$ (in our case $\omega=50$ ) be a sequence of intervals around $i$, decreasing from $[0, t]$ to $\{i\}$ :

$$
\begin{gathered}
I_{0}^{i}=[0, t], \\
\forall x, y \in[0, \omega], x<y \Rightarrow I_{y}^{i} \subset I_{x}^{i}, \\
I_{\omega}^{i}=[i, i] .
\end{gathered}
$$

The lenght of an interval $I$ will be denoted by $|I|$. For each $i \in[0, t]$ let $\left\{s_{i, k}^{m r a}\right\}_{k=0}^{\omega}$ and $\left\{s_{i, k}^{m r i}\right\}_{k=0}^{\omega}$ be the averaged sequences over $I_{k}^{i}$ :

$$
s_{i, k}^{\alpha}=\frac{1}{\left|I_{k}^{i}\right|} \sum_{m \in I_{k}^{i}} s_{m}^{\alpha},
$$

In the following, for $i \in[0, t]$ the result of the segmentation of $s_{i}^{\alpha}$ will be denoted $b_{i}^{\alpha}$. Similarly, for $i \in[0, t]$ and $k \in[0, \omega]$, the result of the segmentation of $s_{i, k}^{\alpha}$ will be denoted $b_{i, k}^{\alpha}$. Then we will obtain:

$$
b_{i, \omega}^{\alpha}=b_{i}^{\alpha} .
$$

General description. For each slice $s_{i}^{m r a}$, we have defined two sequences $\left\{s_{i, k}^{m r a}\right\}_{k=0}^{\omega}$ and $\left\{s_{i, k}^{m r i}\right\}_{i=0}^{\omega}$. These sequences start respectively from an average image of all the MRA and MRI slices and finally come respectively to the $i$-th MRA and MRI slice. Assuming that both sequences will smoothly converge from an average image to the current slice, we propose the following segmentation strategy based on an iterative process:

1 initial segmentation of $s_{i, 0}^{m r a}$ and $s_{i, 0}^{m r i}$;

2 for $k=1$ to $\omega$ :

(a) segmentation of $s_{i, k}^{m r i}$, using $b_{i, k-1}^{m r a}$ and $b_{i, k-1}^{m r i}$;

(b) segmentation of $s_{i, k}^{m r a}$, using $b_{i, k-1}^{m r a}$ and $b_{i, k}^{m r i}$.

The process starts from average images and iteratively uses previous segmentations of both modalities to carry out the current segmentation. This current segmentation is carried out by using a watershed algorithm while previous segmentations are used for creation of markers dedicated to watershed initialization. In the following paragraphs, the segmentation process is more precisely described. The gradient computation and template creation steps are explained in specific paragraphs. 


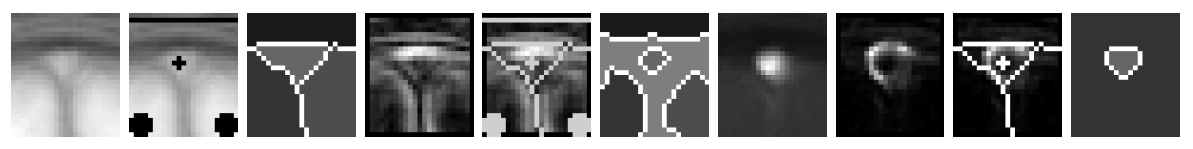

Figure 2. Initialization process. From left to right: MRI average slice $\left(s_{i, 0}^{m r i}\right)$; MRI with four markers; first segmentation; MRI gradient; MRI gradient with five markers; final MRI segmentation $\left(b_{i, 0}^{m r i}\right)$; MRA average slice $\left(s_{i, 0}^{m r a}\right)$; MRA gradient; MRA gradient with two markers; MRA segmentation $\left(b_{i, 0}^{m r a}\right)$.

Initialization. The first step consists in performing the segmentation of $s_{i, 0}^{m r a}$ and $s_{i, 0}^{m r i}$ for $i \in[0, \omega]$. By definition, for all $i, j \in[0, \omega], s_{i, 0}^{\alpha}=s_{j, 0}^{\alpha}$. Thus the initialization step only requires to segment two average slices, one for the MRA and the MRI. The initialization is organized as follows (Figure 2):

1 grey-level opening of the MRA slice with a flat structuring element $(3 \times 3$ cross): the points of maximum value become the markers for the flowing blood in the MRA and MRI slices;

2 successive grey-level openings of the MRI slice with three flat structuring elements (a one voxel width line and two $5 \times 5$ circular elements): the points of maximum value become the markers for the brain and the bone in the MRI slices;

3 watershed segmentation of the MRI slice using the four markers;

4 gradient computation of the MRI slice and watershed segmentation of the gradient MRI slice using the four markers plus a new marker provided by the frontier between the four regions of the previous segmentation;

5 gradient computation of the MRA slice and watershed segmentation of the gradient MRA slice using one marker plus a new marker provided by the frontier between the four regions of the first MRI segmentation.

Standard shape and size of the different structuring elements have been chosen in order to fit the different structures to find. That step finally provides $b_{i, 0}^{m r i}$ and $b_{i, 0}^{m r a}$ then enabling to initialize the iterative process for each $i \in[0, t]$.

Iterative process. For each $i \in[0, t]$, at the step $k$, the iterative process consists in first segmenting the current MRI slice $\left(s_{i, k}^{m r i}\right)$ by using the MRA and MRI segmentation of the previous step $\left(b_{i, k-1}^{m r a}\right.$ and $\left.b_{i, k-1}^{m r i}\right)$. Then the current MRA slice can be segmented using the current MRI segmentation $\left(b_{i, k}^{m r i}\right)$ and the previous MRA segmentation $\left(b_{i, k-1}^{m r a}\right)$. A step calculating $b_{i, k}^{m r a}$ and $b_{i, k}^{m r i}$ can be decomposed as follows (Figure 3): 

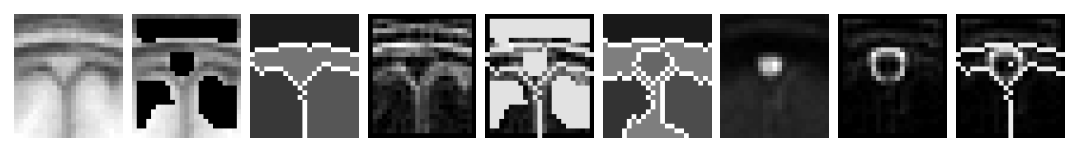

Figure 3. A step of the iterative process for one slice. From left to right: MRI average slice $\left(s_{i, k}^{m r i}\right)$; MRI with four markers; first segmentation; MRI gradient; MRI gradient with five markers; final MRI segmentation $\left(b_{i, k}^{m r i}\right)$; MRA average slice $\left(s_{i, k}^{m r a}\right)$; MRA gradient; MRA gradient with two markers; MRA segmentation $\left(b_{i, k}^{m r a}\right)$.

1 creation of four markers to initialize $s_{i, k}^{m r i}$ segmentation;

2 watershed segmentation of $s_{i, k}^{m r i}$, using the four markers;

3 gradient computation of $s_{i, k}^{m r i}$ and watershed segmentation of $s_{i, k}^{m r i}$ gradient, using the four markers plus one marker provided by the frontier between the four regions found by the previous watershed segmentation;

4 creation of one marker to initialize $s_{i, k}^{m r a}$ segmentation;

5 gradient computation of $s_{i, k}^{m r a}$ and watershed segmentation of $s_{i, k}^{m r a}$ gradient, using the marker plus one marker provided by the frontier between the four regions found by the first watershed segmentation of $s_{i, k}^{m r i}$;

Gradient computation. For each step, the segmentation of both MRA and MRI slices requires the computation of gradient images. Concerning the MRI slices, the gradient is computed by choosing the maximum intensity variation in the four principal directions, then correctly delineating the four main structures from the low intensity regions. This gradient calculation gives correct results as the four regions of interest have homogeneous intensity levels. This is not the case of the flowing blood in the MRA slices. Indeed, the blood present a very high but heterogeneous level. Since computing a simple gradient does not allow to obtain well defined frontiers, a solution consists in dividing the gradient value calculated at a pixel by the pixel value. This normalized gradient will present low values in homogeneous high intensity regions and high values for the background points located at the frontier with the flowing blood.

Markers generation. At each step of the iterative process and for each slice, it is necessary to generate markers to initialize the watershed segmentations. The segmentation of the MRI slice requires four markers: one for the dura mater, one for both hemispheres and one for the skull. For any MRI slice $s_{i, k}^{m r i}$, the markers are generated as follows. First, three templates, for hemispheres and skull are created from $s_{i, k-1}^{m r i}$. They are obtained by performing a greylevel erosion with a flat structuring element $(3 \times 3$ square $)$ on $s_{i, k-1}^{m r i}$. For each 
template, $b_{i, k-1}^{m r i}$ is used as a mask to indicate what are the regions where the erosion has to be performed. A fourth template, for the dura mater, is obtained by performing a grey-level erosion with a flat structuring element $(3 \times 3$ cross $)$ on $s_{i, k-1}^{m r i}$. Both $b_{i, k-1}^{m r i}$ and $b_{i, k-1}^{m r a}$ are used as mask to indicate what are the regions where the erosion has to be performed. Then, for each template a grey-level erosion of $s_{i, k}^{m r i}$ using the current template as a grey-level structuring element is performed. After the erosion, a dilation using the template as a binary element is carried out at the maximum point of the eroded image, then providing a marker for watershed initialization.

The segmentation of the MRA slice requires one marker indicating the position of the flowing blood. For any MRA slice $s_{i, k}^{m r a}$, the marker is choosen as being the pixel of maximal intensity in the region of $s_{i, k}^{m r a}$ delimited by the vascular region segmented in $b_{i, k-1}^{m r a}$.

Postprocessing. The segmentation provides two sets of slices. The first gives the segmentation of the flowing blood in the MRA set while the second gives the segmentation of the sinus blood and wall in the MRI set. Then, these slices have to be put back to their correct position in the initial images. During this step, it might happen that small gaps appear between successives slices. Since these gaps are quite small (their thickness is never larger than one voxel), they can then be filled by using a binary closing with a linear structuring element composed of 3 voxels and oriented according to the direction of the axis. As a very last step, the user can also choose to apply a binary opening to smooth the image (we propose here to use a $3 \times 3 \times 3$ cross).

\section{Experimental section}

Data acquisition. A database of 6 patients has been used to validate the efficiency of the proposed algorithm. For each patient, two MR data have been provided (Figure 1): a T1 MRI of the whole head $(280 \times 240 \times 256$ voxels) and a MRA of the top of the head $(192 \times 70 \times 256$ voxels $)$. Voxels are cubes of $1 \mathrm{~mm}$ edge.

Complexity and computation time. The proposed algorithm has a complexity of $O(\omega . t . x . y . m)$, where $\omega$ is the number of iterations, $t$ is the number of slices, $x$ and $y$ are the slice dimensions and $m$ is the maximum area of all the used structuring elements $(m \ll x . y)$. The images have been segmented with a computer using a $2.4 \mathrm{GHz}$ Pentium IV processor with $2 \mathrm{~GB}$ of memory. The average computation time is then 6 minutes. It has to be noticed that the proposed algorithm runs in an entirely automatic fashion. 

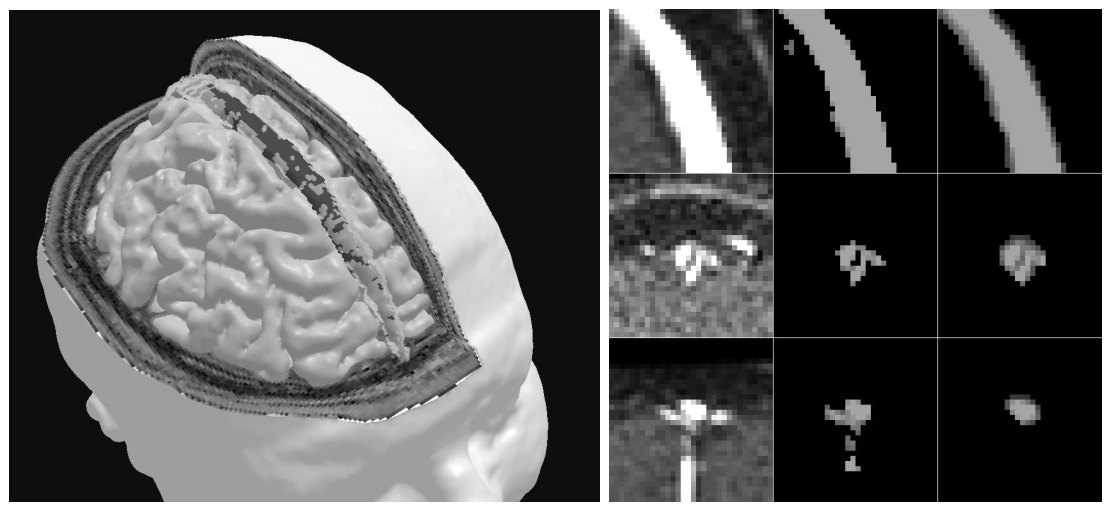

Figure 4. Left: flowing blood surrounded by the SSS wall. Right: results provided by the proposed method and a region growing method. First column: MRA data. Second column: region growing segmentation. Third column: proposed segmentation.

\section{Results and discussion}

The results obtained with the proposed method have been compared to those provided by a region growing algorithm proposed in [8]. All the validations have been carried out by a human specialist who qualitatively tested both algorithms on each case of the previously described database.

During the validation, it has been observed that the method could segment the flowing blood even when MRA signal was heterogeneous or low. The right pictures of Figure 4 illustrate the main observations of the validations. For highly homogeneous intensity regions, it has been observed that both algorithms provide correct segmentations. Nevertheless, the region growing algorithm is sensitive to aliasing artefacts while the proposed algorithm is more robust, also segmenting the low intensity flowing blood in the middle of the vessel. The proposed algorithm is also able to segment only the sinus while the region growing algorithm also segments connected veins.

\section{Conclusion}

This paper presents a novel method, based on watershed segmentation and mathematical morphology operators guided by anatomical knowledge. This method is dedicated to SSS segmentation from brain multimodal MR data. It has been tested on 6 cases, providing more precise results than a previously proposed region growing algorithm, even in case of strong inhomogeneity of signal in MRA data. The main originality of this work consists in integrating high level anatomical knowledge, and using both MRA and MRI data in order to guide mathematical morphology tools. A first attempt to integrate anatom- 
ical knowledge in a vessel segmentation process had already been proposed in [8], where an atlas was used to divide the brain into areas having homogeneous vascular properties. The method proposed here can be considered as being dedicated to one of these areas (the SSS area), then proposing a reliable strategy for the vessels it contains. This work makes part of a new kind of segmentation strategies consisting in processing each part of a vascular tree in a adapted fashion, instead of processing all the vessels in a global way. Further work will now consist in using this method as a first step for segmentation and topology recovery of the whole cerebral superficial venous tree.

\section{Acknowledgements}

The authors thank the EPML \#9 CNRS-STIC for its financial support. They also thank C. Maillot for his precious knowledge on human anatomy.

\section{References}

[1] S.R. Aylward and E. Bullit. Initialization, noise, singularities, and scale in height ridge traversal for tubular object centerline extraction. IEEE Transactions on Medical Imaging, 21:61-75, 2002.

[2] S. Beucher and F. Meyer. The morphological approach to segmentation: the watershed transformation, chapter 12, pages 433-481. E.R. Dougherty, Ed. Marcel Dekker, 1993.

[3] H.E. Cline, D.R. Thedens, C.H. Meyer, D.G. Nishimura, T.K. Foo, and S. Ludke. Combined connectivity and a grey-level morphological filter in magnetic resonance coronary angiography. Magnetic Resonance in Medicine, 43:892-995, 2000.

[4] Y.P. Du and D.L. Parker. Vessel enhancement filtering in three-dimensional angiograms using long-range signal correlation. Journal of Magnetic Resonance Imaging, 7:447-450, 1997.

[5] C.L. Dumoulin and H.R. Hart. Magnetic resonance angiography. Radiology, 161:717$720,1986$.

[6] N. Flasque, M. Desvignes, J.M. Constans, and M. Revenu. Acquisition, segmentation and tracking of the cerebral vascular tree on 3D magnetic resonance angiography images. Medical Image Analysis, 5:173-183, 2001.

[7] G. Gerig, T. Koller, G Székely, C. Brechbühler, and O. Kübler. Symbolic description of 3-D structures applied to cerebral vessel tree obtained from MR angiography volume data. In IPMI'93, volume 687 of LNCS, pages 94-111, 1993.

[8] N. Passat, C. Ronse, J. Baruthio, J.-P. Armspach, C. Maillot, and C. Jahn. Atlas-based method for segmentation of cerebral vascular trees from phase-contrast magnetic resonance angiography. In SPIE Image Processing 2004, volume 5370, pages 420-431, 2004.

[9] A.R. Sanderson, D.L. Parker, and T.C. Henderson. Simultaneous segmentation of MR and X-ray angiograms for visualization of cerebral vascular anatomy. In VIP'93, 1993.

[10] P.J. Yim, P.L. Choyke, and R.M. Summers. Gray-scale skeletonization of small vessels in magnetic resonance angiography. IEEE Transactions on Medical Imaging, 19:568-576, 2000 .

[11] C. Zahlten, H. Jürgens, C.J.G. Evertsz, R. Leppek, H.-O. Peitgen, and K.J. Klose. Portal vein reconstruction based on topology. European Journal of Radiology, 19:96-100, 1995. 